\title{
APLICAÇÃO DO ÍNDICE DE VEGETAÇÃO NDVI (NORMALIZED DIFFERENCE VEGETATION INDEX) EM IMAGENS DE ALTA RESOLUÇÃO NO MUNICÍPIO DE SÃO PAULO E SUAS LIMITAÇÕES
}

\author{
Giuliana Del Nero Velasco ${ }^{1}$, Jefferson Lordello Polizel ${ }^{2}$, Priscila Pereira Coltri ${ }^{3}$, Ana Maria \\ Liner Pereira Lima ${ }^{4}$, Demóstenes Ferreira da Silva Filho ${ }^{5}$
}

\begin{abstract}
RESUMO
O objetivo do trabalho foi testar a aplicação do índice de vegetação NDVI em imagens de alta resolução, em toda a área do município de São Paulo. Foram utilizadas imagens do satélite IKONOS II. O programa de geoprocessamento utilizado foi o TNT Mips 6.8, da Microlmages. O índice aplicado foi o NDVI (Normalized Difference Vegetation Index), que tem como variáveis as bandas do vermelho e infravermelho próximo. Notou-se, com a obtenção do índice, uma desigualdade de cores, sendo nítida a presença de faixas que não deveriam ter ocorrido. Tal fato demonstrou a ocorrência de algum tipo de erro, o que impedia a obtenção de um índice que representasse, fielmente, todo o município. Isso ainda é reforçado pelo fato do NDVI ser interpretado por tonalidade de cores, ou seja, a variação na presença de vegetação deve ser representada por uma diferença de cor; um erro gerado no índice, resultando em diferentes faixas de tonalidade, poderia prejudicar a correta interpretação do mesmo. Concluiu-se que, para o Município de São Paulo, com as imagens supracitadas, deve-se atentar quando da aplicação do índice de vegetação NDVI, não sendo possível analisá-lo em toda a extensão do município, mas sim, apenas dentro das faixas específicas geradas.
\end{abstract}

Palavras-Chave: índice de vegetação, arborização urbana, sensoriamento remoto.

\footnotetext{
1. Engenheira Agrônoma, doutoranda, ESALQ/USP. Piracicaba-SP, gdnvelas@esalq.usp.br.

2. Analista de Sistema, mestrando em geografia, FFLCH/USP. São Paulo-SP, jlpolize@esalq.usp.br

3. Engenheira agrônoma, bolsista CNPQ, Centro de Previsão de Tempo e Estudos Climáticos, CPTEC/INPE, pcoltri@cptec.inpe.br

4. Engenheira agrônoma, Profa. Dra. Depto. Produção Vegetal, ESALQ/USP. Piracicaba-SP

${ }^{5}$. Engenheiro Agrônomo, Prof. Dr. Depto. de Ciências Florestais, ESALQ/USP. Piracicaba-SP, dfsilva@esalq.usp.br
} 


\title{
APPLICATION OF THE INDEX OF VEGETATION NDVI (NORMALIZED DIFFERENCE VEGETATION INDEX) IN HIGH RESOLUTION IMAGES IN THE CITY OF SÃO PAULO AND ITS LIMITATIONS
}

\begin{abstract}
The objective of the work was to test the application of the index of high resolution vegetation NDVI in images, in all the area of the city of Sao Paulo. The work was developed with the use of images of satellite IKONOS II. The program used was TNT MIPS 6,8 , of the Microlmages. The applied index of vegetation was NDVI (Normalized Difference Vegetation Index), that it consists of an equation that has as changeable the bands of the red and next infra-red ray. It was noticed, with the attainment of the index, an inaquality of colors, being clear the presence of bands that must not have occurred. Such fact demonstrated occurrence of some type of error, what it hindered the attainment of an index that represented, faithful, all the city, from the supplied images. This still is strengthened by the fact of the NDVI to be interpreted by tonality of colors, that is, the variation in the vegetation presence must be represented by a color difference; an error generated in the index, resulting in different bands of tonality, could harm the correct interpretation of the same. One concluded that, with the images of available satellite, must be attempted against when of the application of the index of vegetation NDVI, not being possible to analyze it in all the extension of the city, but yes, only inside of the generated specific bands.
\end{abstract}

Key-words: vegetation index, urban arborization, remote sensing 


\section{INTRODUÇÃO}

O uso de imagens de satélite para estudos de vegetação no ambiente urbano é uma prática muito utilizada principalmente quando se trata de cidades de grande porte, como é o caso do município de São Paulo, com $1.523 \mathrm{~km}^{2}$ de extensão, segundo dados do IBGE (2006). Segundo Florenzano (2002), as imagens de satélite proporcionam uma visão sinóptica (de conjunto) e multitemporal (de dinâmica) de extensas áreas da superfície terrestre. Assim, as imagens de satélite da área permitem uma visão do todo, possibilitando a obtenção de informações que seriam inviáveis de serem adquiridas sem a utilização das mesmas.

O satélite Ikonos II, considerado de altíssima resolução espacial (1m na pancromática e 4m na multiespectral), foi colocado na órbita em 24 de setembro de 1999 a uma altitude de $680 \mathrm{~km}$, operando nos comprimentos de onda entre 400 e $1.100 \mathrm{~nm}$. (Moreira, 2005).

O mesmo autor afirma que cada alvo (asfalto, vegetação, água, entre outros) tem comportamento espectral diferente na mesma condição ambiental. A água apresenta a mais baixa reflectância e absorve toda a radiação acima de $750 \mathrm{~nm}$. O asfalto não ultrapassa $10 \%$ no valor da reflectância. Segundo Tucker, (1979), citado por Moreira (2005), a vegetação é caracterizada por uma intensa absorção devido à clorofila na região do vermelho $(0,58$ a $0,68 \mu \mathrm{m})$ e por uma intensa reflexão na faixa do infravermelho próximo $(0,76$ a 1,35 $\mu \mathrm{m})$ causada pela estrutura celular das folhas. A diferença entre as bandas do vermelho e infravermelho é proporcional à reflectância da imagem, sendo a medida do grau de vegetação na imagem. Moreira (2005) afirma que os dados de reflectância dos alvos podem ser transformados em índices de vegetação, os quais foram criados com o intuito de ressaltar o comportamento espectral da vegetação em relação ao solo e a outros alvos da superfície terrestre, sendo que um dos índices mais utilizados é o NDVI (Normalized Difference Vegetation Index).

O objetivo do presente trabalho foi testar a aplicação do índice de vegetação NDVI em imagens de alta resolução, em toda a área do município de São Paulo.

\section{MATERIAL E MÉTODOS}

Foram utilizadas imagens do satélite IKONOS II, cedidas pelo Instituto Geológico, vinculado à Secretaria do Meio Ambiente do Governo do Estado de São Paulo. As imagens foram tiradas no ano de 2002 e cada cena comporta uma área de $5 \times 5 \mathrm{~km}$. Foram fornecidas no formato geotiff nas bandas azul (blue), vermelho (red), verde (green), infravermelho próximo (NIR) e na composição vermelho-verde-azul (RGB). 
Para abranger todo o município de São Paulo, foram necessárias 67 cenas do satélite. O programa de geoprocessamento utilizado para realização da presente etapa da pesquisa foi o TNT Mips 6.8, da Microlmages.

Para a obtenção do índice, as seguintes etapas foram seguidas:

\section{Geração de mosaicos}

Foram gerados dois mosaicos, um da banda RED e um da NIR, os quais consistiram em um agrupamento das 67 cenas correspondentes à área do Município, tal qual um quebra-cabeça, resultando em um mosaico composto por uma só cena englobando todo o município. A etapa foi feita utilizando as ferramentas "process", "raster", "mosaic" do programa utilizado.

\section{Aplicação do Índice de vegetação:}

O índice de vegetação utilizado no presente trabalho foi o NDVI (Normalized Difference Vegetation Index), o qual consiste em uma equação que tem como variáveis as bandas do vermelho e infravermelho próximo, como se segue:

NDVI: IVP $-V / I V P+V$

Onde,

IVP: valor da reflectância da banda no Infravermelho próximo

V: valor de reflectância da banda no vermelho

O índice também foi obtido pelo programa TNT Mips através das etapas "process", "raster", "combine", "predefined".

\section{Análise do índice e de suas limitações}

Nesta etapa foi analisado o índice de vegetação e seus problemas e limitações, tentando corrigi-los.

\section{RESULTADOS E DISCUSSÃO}

O mosaico das 67 cenas das bandas NIR e RED podem ser vistos nas figuras 1 e 2 , respectivamente. 


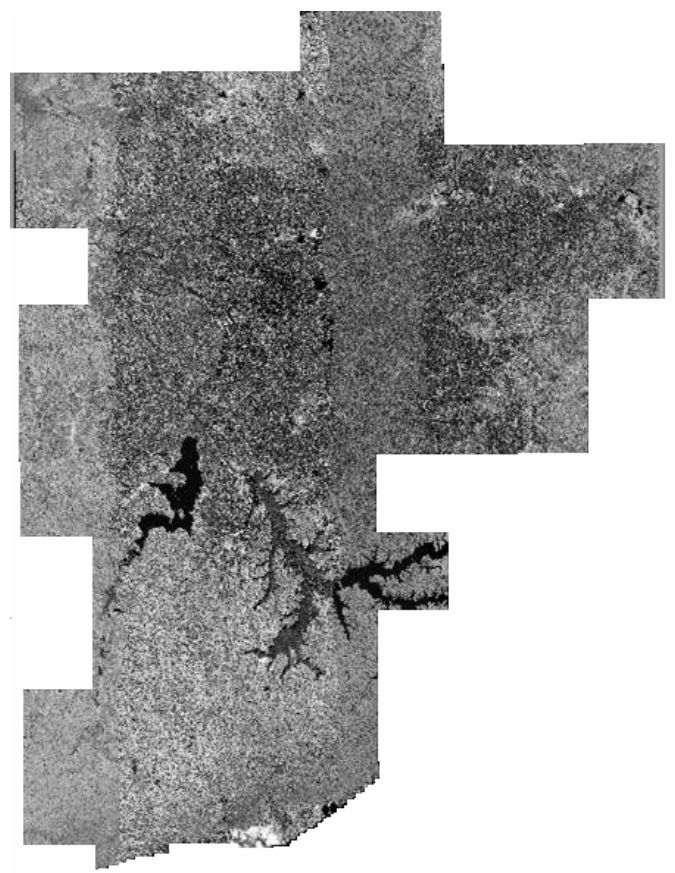

Figura 1. Mosaico das 67 cenas da banda NIR compreendendo o Município de São Paulo.

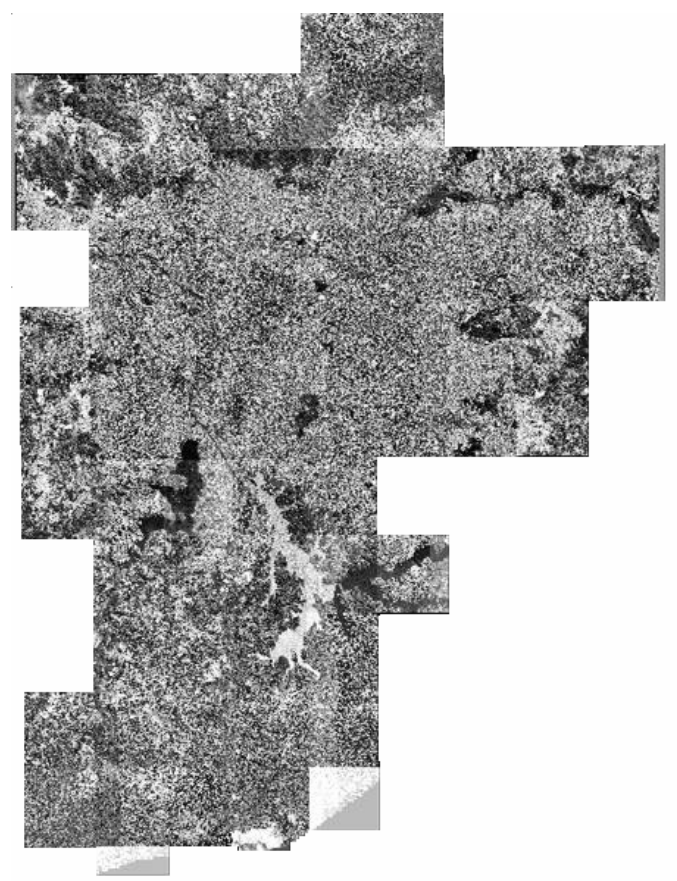

Figura 2. Mosaico das 67 cenas da banda RED compreendendo o Município de São Paulo. 
O índice de vegetação gerado pode ser visto na figura 3.

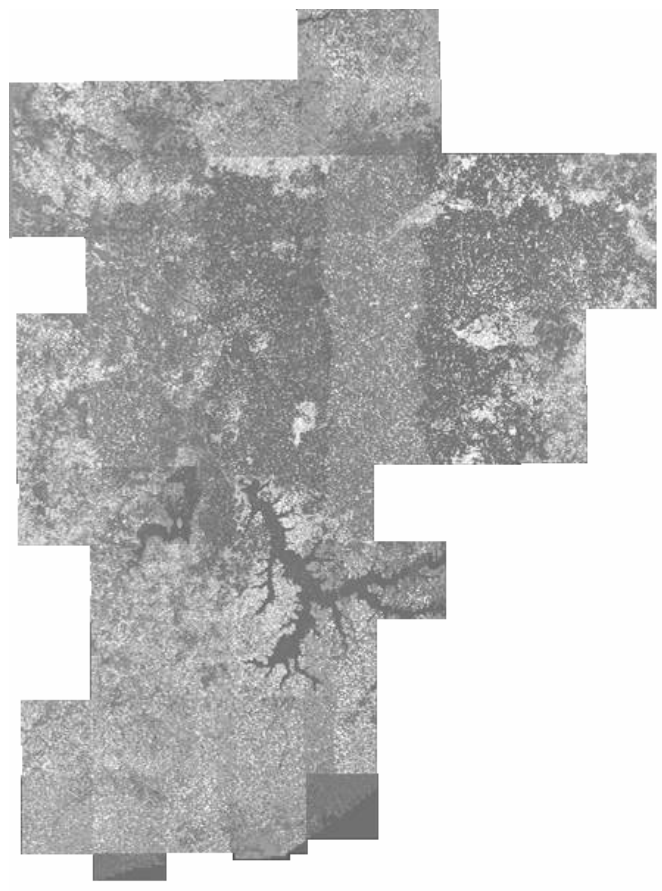

Figura 3. Índice de vegetação (NDVI).

A figura 4 mostra o NDVI e o limite que confere o Município de São Paulo.

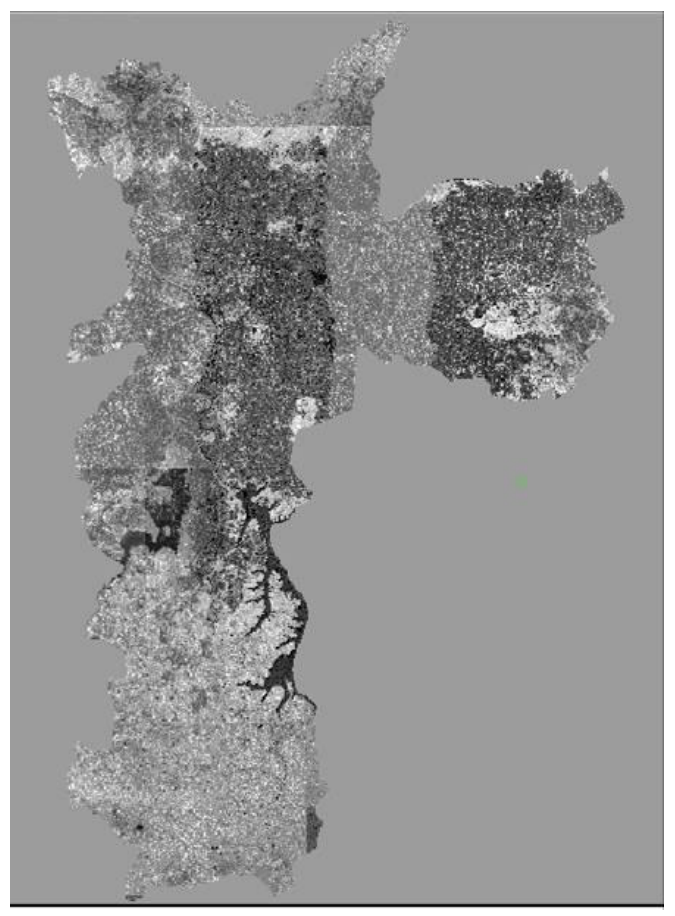

Figura 4. Índice de vegetação NDVI com limite do Município de SP 
Notou-se com a obtenção do índice, uma desigualdade de cores, sendo nítida a presença de faixas verticais que não deveriam ter ocorrido, já que dentro de faixas de cores diferentes, quando estudadas separadamente, ocorriam os mesmos tipos de vegetação. Tal fato demonstrou a ocorrência de algum tipo de erro o que impedia a obtenção de um índice que representasse, fielmente, todo o município. Isso ainda é reforçado pelo fato do NDVI ser interpretado por tonalidade de cores, ou seja, a variação na presença de vegetação deve ser representada por uma diferença de cor; um erro gerado no índice resultando em diferentes faixas de tonalidade poderia prejudicar a correta interpretação do mesmo.

Com isso, iniciou-se uma tentativa de corrigir essa diferença na tonalidade do índice através de ferramentas fornecidas pelo software utilizado. Os mosaicos foram gerados novamente, bem como o índice, para conferir se o procedimento tinha sido feito de maneira correta, mas não houve diferença no resultado. Também foram feitos diversos testes com a geração dos mosaicos e a aplicação de filtros de normalização e equalização ("normalize" e "equalize"), no intuito de homogeneizar a tonalidade, mas os resultados não foram positivos. Assim, as cenas foram estudadas individualmente e descobriu-se que a diferença de tonalidade estava dentro de cada cena, ou seja, grande parte das cenas fornecidas estava com essa diferença de cor. Uma possível explicação para esse problema é a de que as cenas foram obtidas no ano de 2002 e algumas foram corrigidas no ano seguinte, podendo gerar tal diferença.

Com essa constatação, e com a impossibilidade de obter-se outras cenas, procurouse corrigir aquelas que apresentavam tal problema, uma a uma, para assim gerar novamente os mosaicos e em seguida o índice de vegetação.

Assim, somando as bandas do vermelho e infravermelho, totalizavam 134 cenas a serem corrigidas. O procedimento feito foi o de, cena a cena, dividi-la em dois pedaços, exatamente no limite onde estava a diferença de coloração e então gerar novo mosaico dos dois pedaços, com a aplicação de um filtro de equalização ("equalize") no intuito de homogeneizar a tonalidade dentro de cada cena.

Um exemplo de uma cena que apresenta diferença na tonalidade pode ser visto na figura 5 . 


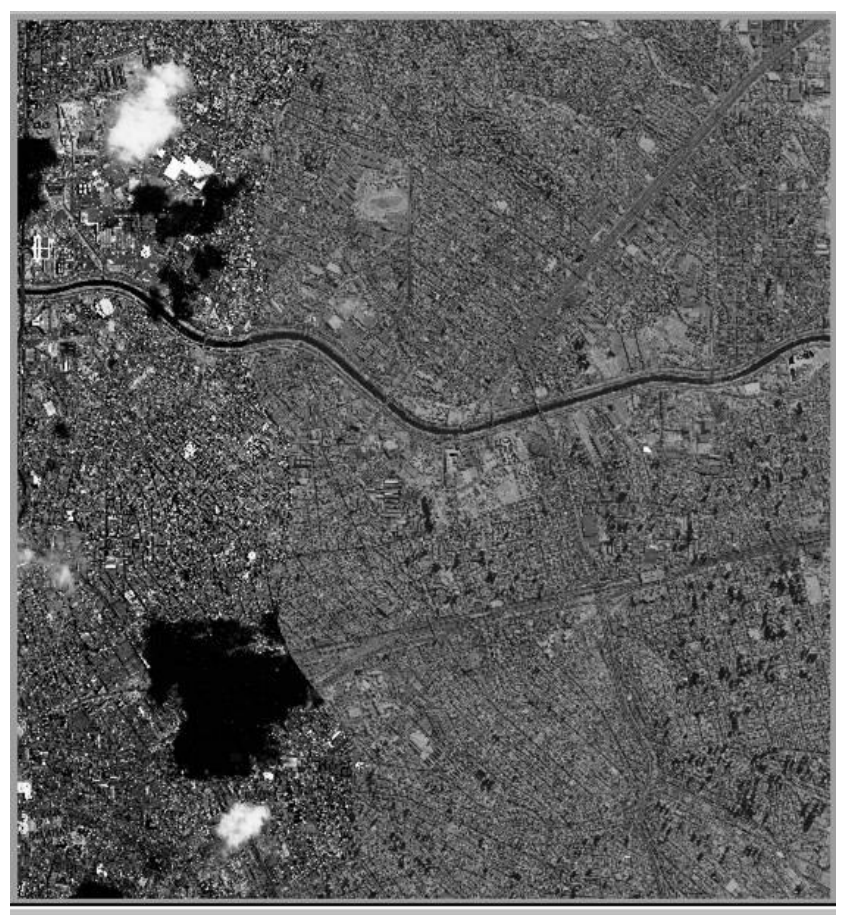

Figura 5. Cena NIR mostrando diferença na tonalidade.

A figura 6 mostra a cena dividida em dois polígonos exatamente no limite em que ocorre a diferença de tonalidade.
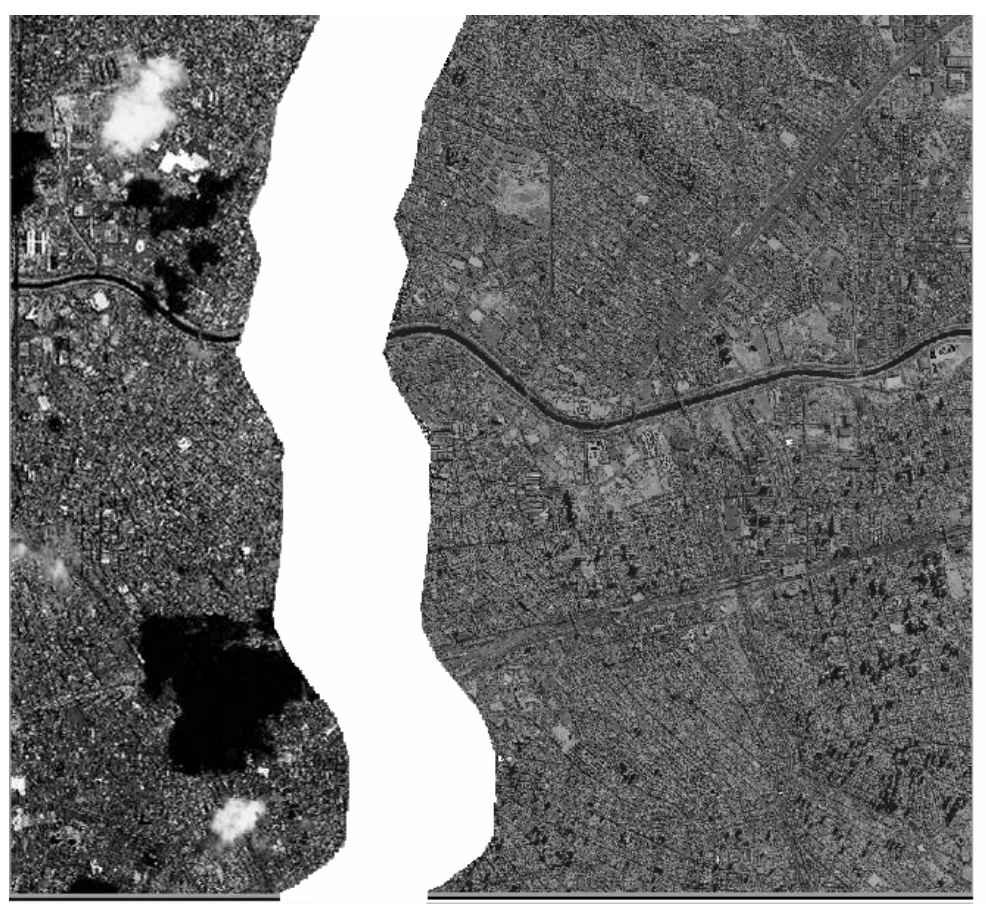

Figura 6. Cena cortada na região da diferença de tonalidade. 
Depois de cortada em dois pedaços, as cenas foram novamente unidas, agora com o uso do filtro "equalize". A figura 7 mostra a cena recomposta já com a aplicação do filtro.

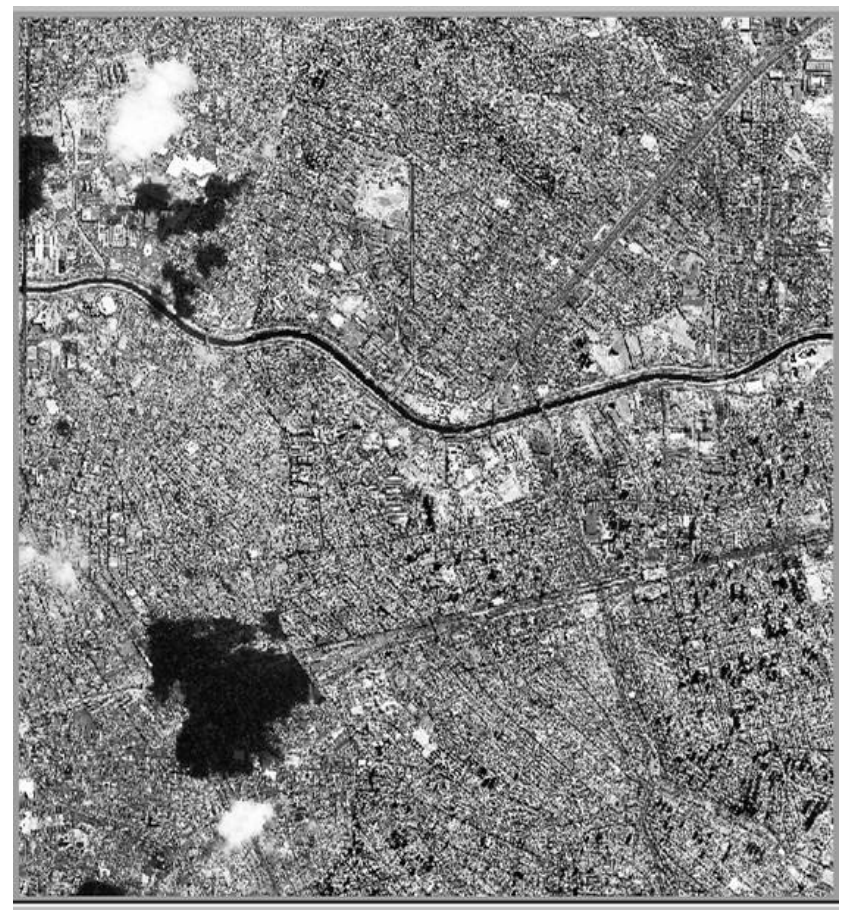

Figura 7. Cena mosaicada com uso do filtro "equalize".

Notou-se com a aplicação do filtro que a cena ficou mais homogênea em termos de coloração, embora ainda fosse possível notar diferença de cor. Assim, foi feito o mesmo procedimento nas 134 cenas, ou seja, cada cena foi dividida em dois pedaços e estes unidos com o uso do filtro "equalize", voltando a ser uma só cena. Em seguida foi feito o mosaico das 67 cenas do NIR e das 67 cenas do RED, resultando novamente em duas cenas (uma de cada banda) abrangendo o Município de SP, agora equalizadas.

Os mosaicos já equalizados das bandas NIR e RED podem ser vistos nas figuras 8 e 9, respectivamente. 


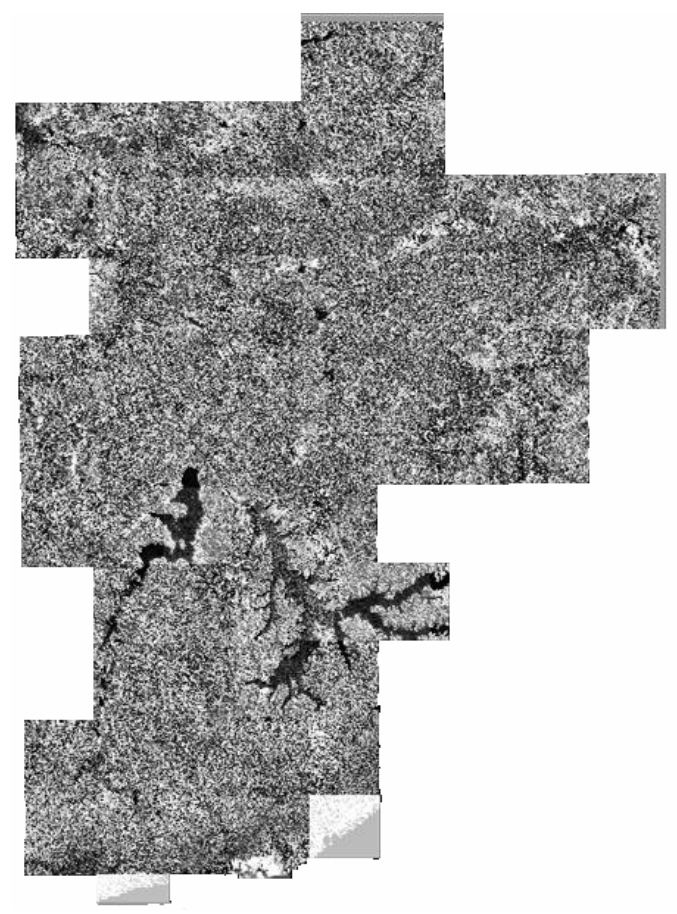

Figura 8. Mosaico NIR equalizado.

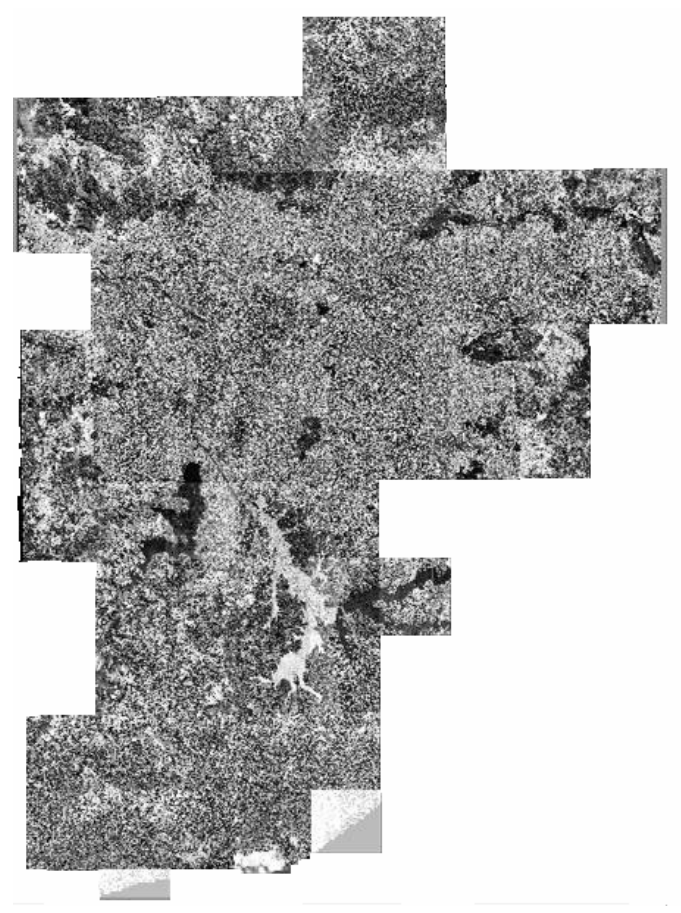

Figura 9. Mosaico Red equalizado.

A etapa seguinte foi aplicar o índice NDVI com o uso dos mosaicos NIR e RED equalizados. O resultado do índice pode ser visto na figura 10. 


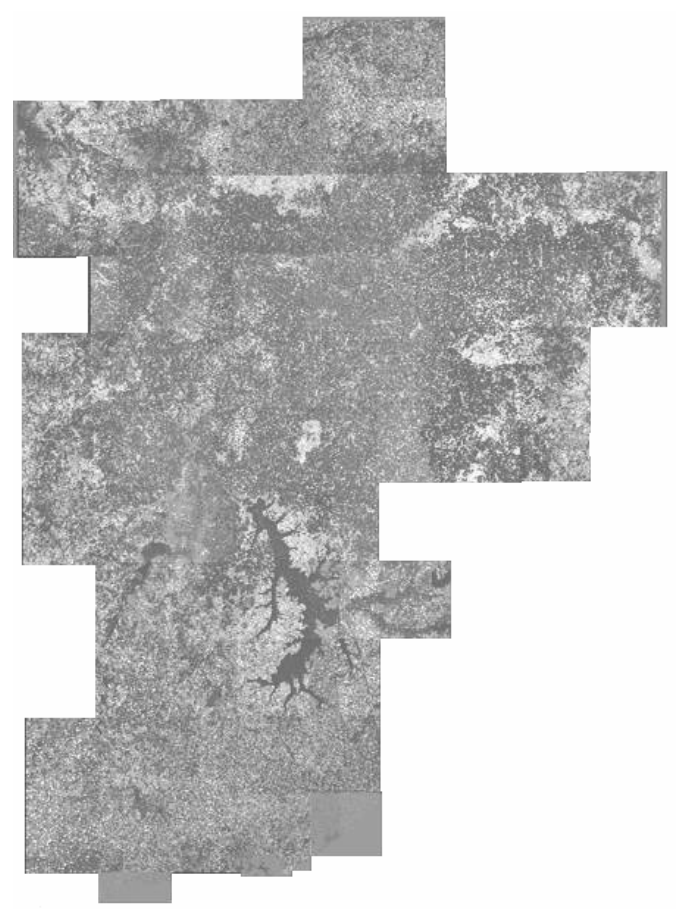

Figura 10. Índice NDVI equalizado.

Notou-se com a figura que a aplicação do filtro "equalize" não solucionou os problemas de diferença de tonalidade: embora tenha havido uma relativa homogeneização das faixas anteriormente vistas, notou-se uma fragmentação das cenas, sendo possível identificar as 67 cenas, o que não ocorre quando o resultado do índice é correto.

Cabe ressaltar que as imagens do satélite IKONOS que foram utilizadas para gerar tal índice são as únicas existentes para o município de São Paulo, sendo as mesmas utilizadas para outros estudos. O presente estudo poderá servir de base para futuros trabalhos, principalmente pela detecção da presença de tal erro. Futuros estudos já podem partir do princípio que o índice de vegetação NDVI com imagens do satélite IKONOS s'pode ser utilizado em partes, dentro de cada faixa, não podendo ser analisado de forma homogênea em todo o município.

\section{CONCLUSÕES}

Concluiu-se que, para Município de São Paulo, com as imagens do satélite IKONOS disponíveis, deve-se atentar quando da aplicação do índice de vegetação NDVI, não sendo possível analisá-lo em toda a extensão do município, mas sim, apenas dentro das faixas específicas geradas. 


\section{AGRADECIMENTOS}

Os autores agradem à FAPESP - Fundação de Amparo à Pesquisa do Estado de São Paulo pela concessão da bolsa de doutorado no qual o presente estudo está inserido.

\section{REFERÊNCIAS BIBLIOGRÁFICAS}

Florenzano, T. G. Imagens de satélite para estudos ambientais. Oficina de textos, 2002. São Paulo. 97p.

INSTITUTO BRASILEIRO DE GEOGRAFIA E ESTATÍSTICA- IBGE. Disponível em http://www.ibge.gov.br . Acesso em: 06set. 2006.

Moreira, M. A. Fundamentos do sensoriamento remoto e metodologias de aplicação. $3^{\text {a }}$ edição. 2005. Editora UFV.320p. 